\title{
$\lim ^{m / 2}$
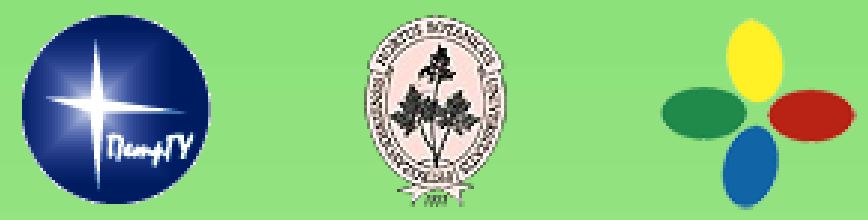 \\ HORTUS BOTANICUS
}

Международный электронный журнал ботанических садов

\section{4 / 2019}

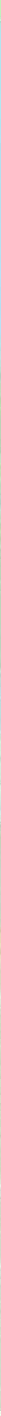


Информационно-аналитический центр Совета ботанических садов России при Ботаническом саде Петрозаводского государственного университета

\section{HORTUS BOTANICUS}

Международный электронный журнал ботанических садов

\section{$14 / 2019$}

ISSN 1994-3849

Эл № ФС 77-33059 от 11.09.2008

\section{Главный редактор}

А. А. Прохоров

Редакционный совет

П. Вайс Джексон Лей Ши

Йонг-Шик Ким

Т. С. Мамедов

В. Н. Решетников
Редакционная коллегия

Г. С. Антипина

Е. М. Арнаутова

А. В. Бобров

Ю. К. Виноградова

Е. В. Голосова

Е. Ф. Марковская

Ю. В. Наумцев

Е. В. Спиридович

К.Г. Ткаченко

А. И. Шмаков

\section{Редакция}

Е. А. Платонова

С. М. Кузьменкова

К. О. Романова

А. Г. Марахтанов

\section{Адрес редакции}

185910, Республика Карелия, г. Петрозаводск, ул. Красноармейская, 31, каб. 12.

E-mail:hortbot@gmail.com

http://hb.karelia.ru

(C) 2001-2019 A. А. Прохоров

\section{На обложке:}

Ботанический сад Соловецкого историко-архитектурного музея-заповедника. Врата. Фото Михаила Щеглова.

\section{Разработка и техническая поддержка}

Отдел объединенной редакции научных журналов ПетрГУ, РЦ НИТ ПетрГУ, Ботанический сад ПетрГУ

Петрозаводск 


\section{Каталог семян Полярно-Альпийского ботанического сада-института № 69}

\begin{tabular}{|c|c|}
\hline $\begin{array}{l}\text { ТРОСТЕНЮК } \\
\text { Надежда Николаевна }\end{array}$ & $\begin{array}{l}\text { Полярно-Альпийский ботанический сад-институт имени Н. А. } \\
\text { Аврорина Кольского научного центра РАН, } \\
\text { Ферсмана, 18A, Апатиты, 184209, Россия } \\
\text { tnn_aprec@mail.ru }\end{array}$ \\
\hline $\begin{array}{l}\text { ВИРАЧЕВА } \\
\text { Любовь Леонидовна }\end{array}$ & $\begin{array}{l}\text { Полярно-Альпийский ботанический сад-институт имени Н. A. } \\
\text { Аврорина Кольского научного центра РАН, } \\
\text { Ферсмана, 18A, Апатиты, } 184209, \text { Россия } \\
\text { viracheva-ljubov@yandex.ru }\end{array}$ \\
\hline $\begin{array}{l}\text { ГОНЧАРОВА } \\
\text { Оксана Александровна }\end{array}$ & $\begin{array}{l}\text { Полярно-Альпийский ботанический сад-институт имени Н. A. } \\
\text { Аврорина Кольского научного центра РАН, } \\
\text { Ферсмана, 18А, Апатиты, 184209, Россия } \\
\text { goncharovaoa@mail.ru }\end{array}$ \\
\hline $\begin{array}{l}\text { КИРИЛЛОВА } \\
\text { Наталья Руслановна }\end{array}$ & $\begin{array}{l}\text { Полярно-Альпийский ботанический сад-институт имени Н. A. } \\
\text { Аврорина Кольского научного центра РАН, } \\
\text { Ферсмана, 18A, Апатиты, 184209, Россия } \\
\text { knr81@mail.ru }\end{array}$ \\
\hline $\begin{array}{l}\text { ЛИППОНЕН } \\
\text { Ирина Николаевна }\end{array}$ & $\begin{array}{l}\text { Полярно-Альпийский ботанический сад-институт имени Н. А. } \\
\text { Аврорина Кольского научного центра РАН, } \\
\text { Ферсмана, 18A, Апатиты, 184209, Россия } \\
\text { lipponen-in@yandex.ru }\end{array}$ \\
\hline $\begin{array}{l}\text { Ключевые слова: } \\
\text { список семян, Полярно- } \\
\text { Альпийский ботанический } \\
\text { сад-институт, ПАБСИ, } \\
\text { семена, интродукция, Index }\end{array}$ & $\begin{array}{l}\text { Аннотация: Список семян культивируемых растений, } \\
\text { собранных в 2017-2018 гг. в Полярно-Альпийском } \\
\text { ботаническом саду-институте и предлагаемых для } \\
\text { обмена с другими ботаническими садами и } \\
\text { учреждениями. }\end{array}$ \\
\hline
\end{tabular}

Получена: 07 мая 2019 года

Подписана к печати: 31 июля 2019 года

The Polar-Alpine Botanical Garden was founded on August 26, 1931. It is situated in the southern part of the Khibiny Mountains $120 \mathrm{~km}$ north of the Polar circle. Founder and first director of the Garden was N. A. Avrorin.

The garden has the altitude difference from 312 up to $1068 \mathrm{~m}$ above the sea-level. Its territory equals 1670 hectares, where 1250 hectares are used as sanctuary of the local flora.

The seeds are derived from open-pollinated plants. No guarantee can be given either on the purity or the germination of the seeds.

The volume of families is given according to A. L. Takhtajan (1987), the names of the genera 
and species are given in accordance with The Plant List (2013) and S. K. Cherepanov (1995).

\section{Indicationes meteorologicae}

\begin{tabular}{|c|c|}
\hline Temperatura aeris media annua & $-1.5^{\circ} \mathrm{C}$ \\
\hline Mensis frigidissimi (I, II) temperatura aeris media & $-12.8^{\circ} \mathrm{C}$ \\
\hline Mensis calidissimi (YII) temperatura aeris media & $12.6^{\circ} \mathrm{C}$ \\
\hline Amplitudo vacillationes temperaturae & $25.4^{\circ} \mathrm{C}$ \\
\hline Temperatura aeris absoluta maxima & $30.0^{\circ} \mathrm{C}$ \\
\hline Temperatura aeris absoluta minima & $-42.5^{\circ} \mathrm{C}$ \\
\hline Praecipitatio e caelo media annua & $818 \mathrm{~mm}$ \\
\hline Aeris humiditas relativa media annua & $74 \%$ \\
\hline Nubilositas media annua & 7.7 балл \\
\hline
\end{tabular}

\section{EXPLICATIO}

\section{SIGNA ANTE NUMEROS ET NOMINA}

A - $\quad$ Ager experimentalis Horti nostri prope urbis Apatity

* - Semina anni 2017

Sine stellulis - Semina anni 2018

SIGNA ANTE ANNUM

\begin{tabular}{|c|c|}
\hline p. - & Specimina viva (rhizomata vel bulbi vel taleae) a nobis accepta \\
\hline s. - & Plantae in Horto nostro e seminibus enatae \\
\hline c. - & Plantae ex speciminibus quae saltem uno anno colebantur ortae \\
\hline n. - & Plantae ex speciminibus sponte crescentibus ortae \\
\hline t. - & Clones (e taleis) \\
\hline R. 1, 2 . & Reproductia generationis 1,2 etc. plantarum indicatarum in \\
\hline
\end{tabular}

SIGNA POST ANNUM

\begin{tabular}{ll}
\hline $\mathrm{HB}-$ & Hortus botanicus \\
\hline $\mathrm{HBU}-$ & Hortus botanicus universitatis \\
\hline HBP - & $\begin{array}{l}\text { Hortus botanicus principalis Academiae scientiarum Rossium, } \\
\text { Mosqua }\end{array}$
\end{tabular}




\begin{tabular}{ll}
\hline HBIB - & $\begin{array}{l}\text { Hortus botanicus Instituti Botanici nom. V. L. Komarovii } \\
\text { Academiae scientiarum Rossicum, Petropolis }\end{array}$ \\
\hline NPO - & "VILAR" - Institutum plantarum officinalium, Mosqua \\
\hline AAT - & Academia Agriculturae nom. K. A. Timirjasevii, Mosqua \\
\hline DaINIILH - & Dendrarium of Far East Forestry Research Institute \\
\hline Arb - & Arboretum \\
\hline
\end{tabular}

\section{SIGNA LOGORUM NATALIUM UBI SEMINA SEU PLANTAE LECTA SUNT (PRAETER LOCOS REGIONUS MURMANENSIS QUI IN DIVISIONE II INDICANTUR).}

\begin{tabular}{|c|c|c|}
\hline \multicolumn{2}{|l|}{ Alatau - } & $\begin{array}{l}\text { Kazachstan: Alatau Transiliensis in vicinitate urbis Alma-Aty, } \\
\text { prata alpina et subalpina in declivi boreali, 1500-2500 m s. m. } \\
\text { f. Legit anno } 1935-1937 \text { N. V. Degtjarev }\end{array}$ \\
\hline \multicolumn{2}{|l|}{ Altai - } & $\begin{array}{l}\text { Montes Altai. Legerunt anno } 1934 \text { M. Ch. Kaczurin; anno } \\
1964 \text { B. N. Golovkin et L. A. Shavrov; anno } 1969 \text { G. N. } \\
\text { Andreev, B. N. Golovkin, Z. G. Ulle et L. A. Shavrov }\end{array}$ \\
\hline & $1-$ & Pratum alpinum, montis Czibilik, 1800 m s. m. f. \\
\hline & $2-$ & $\begin{array}{l}\text { Pratum subalpinum inter arbores sparsae Pini sibiricae in } \\
\text { declivitate occidentali montis Sujon (vallis fl. Karakol), } 1750 \mathrm{~m} \\
\text { s. m. f. }\end{array}$ \\
\hline & $4-$ & $\begin{array}{l}\text { Steppae pratensis in vicinitate oppidi Gorno-Altajsk atque in } \\
\text { valle fl. Katunj. } 400-600 \mathrm{~m} \mathrm{~s} \text {. m. f. }\end{array}$ \\
\hline \multicolumn{2}{|l|}{ Bakuriani - } & $\begin{array}{l}\text { Georgia (Grusia): in viciniis pagi Bakuriani, in jugi Trialetensis } \\
\text { monte Zchra-Zkaro. Legerunt anno } 1937 \text { N. A. Avrorin et A. N. } \\
\text { Dzhachishvili; anno1939 A. J. Mischkina; anno } 1946 \text { L. J. } \\
\text { Areschkina et A. N. Dzhavachischvili; anno } 1971 \text { V. A. } \\
\text { Vassilenko, B. N. Golovkin et A. P. Gorelova }\end{array}$ \\
\hline \multicolumn{2}{|l|}{ Carpates - } & $\begin{array}{l}\text { Montes Carpatici orientalis. Legerunt anno } 1956 \text { N. M. } \\
\text { Alexandrova, G. N. Andreev, I. V. Vajnagij et A. A. Kalnin; } \\
\text { anno } 1979 \text { G. N. Andreev, I. V. Vajnagij, A. P. Gorelova et L. } \\
\text { A. Kazakov }\end{array}$ \\
\hline & $1-$ & Mons Bliznitsa, regio subalpina $1500-1800$ m s. m. f. \\
\hline & $2-$ & $\begin{array}{l}\text { Montes Menczul et Scheschul, ad limites superiores } \\
\text { fagetorum, } 1100-1300 \mathrm{~m} \mathrm{~s} \text {. m. f. }\end{array}$ \\
\hline \multicolumn{3}{|l|}{ Czukotka - } \\
\hline & $3-$ & $\begin{array}{l}\text { Paeninsula Czukotka. In viciniis pagi Providentiae (64 } 25^{\prime} \text { lat. } \\
\text { bor, } 173^{\circ} 15^{\prime} \text { long. occ.). Legerunt N. A. Avrorin et G. N. } \\
\text { Andreev, anno } 1958\end{array}$ \\
\hline & $4-$ & $\begin{array}{l}\text { Paeninsula Czukotka. In viciniis pagi Egvekinot }\left(66^{\circ} 11^{\prime} \text { lat. }\right. \\
\text { bor., } 179^{\circ} \text { Iong. occ.) declivum lapidosum australe supra } \\
\text { sinum Crucis maris Beringiani. Legerunt N. A. Avrorin et G. N. } \\
\text { Andreev, anno } 1958\end{array}$ \\
\hline \multicolumn{2}{|l|}{ Igarka - } & $\begin{array}{l}\text { In viciniis oppidi lgarka regionis Krasnojarskensis. Legerunt } \\
\text { anno } 1946 \text { N. A. Avrorin et P. M. Medvedev }\end{array}$ \\
\hline
\end{tabular}




\begin{tabular}{|c|c|c|}
\hline \multicolumn{2}{|l|}{ Kamtschatka- } & $\begin{array}{l}\text { Paeninsula Kamtschatka. Legerunt anno } 1982 \text { A. P. Gorelova, } \\
\text { A. Ph. Zajtseva et L. A. Kazakov; anno } 1985 \text { G. N. Andreev }\end{array}$ \\
\hline Sachalin - & & $\begin{array}{l}\text { Pars australis regionis Sachalinensis (insula Sachalin). } \\
\text { Legerunt anno } 1946 \text { N. A. Avrorin, S. M. Kravczenko et P. M. } \\
\text { Medvedev; anno } 1967 \text { N. M. Alexandrova, B. N. Golovkin, M. } \\
\text { L. Ramenskaja et L. A. Schavrov }\end{array}$ \\
\hline \multirow[t]{2}{*}{ Sajany - } & & $\begin{array}{l}\text { In jugo et depressione Tunkensi montium Sajanensium. Legit } \\
\text { anno } 1936 \text { A. A. Korovkin }\end{array}$ \\
\hline & $3-$ & $\begin{array}{l}\text { Pineto-laricetum magniherbosum in declivi australi, } 1600 \text { m s. } \\
\text { m. f. }\end{array}$ \\
\hline \multicolumn{3}{|l|}{ Jakutsk - } \\
\hline & $2-$ & $\begin{array}{l}\text { Pars inundata ancestralis vallis flumninisLenain viciniis urbis } \\
\text { Jacutsk. Legerunt N. A. Avrorin et G. N. Andreev, anno } 1958\end{array}$ \\
\hline & $3-$ & $\begin{array}{l}\text { In viciniis urbis Magadan ( } 57^{\circ} 35^{\prime} \text { lat. bor., } 150^{\circ} 50^{\prime} \text { long. or.). } \\
\text { Legerunt N. A. Avrorin et G. N.Andreev anno } 1958 \text {. }\end{array}$ \\
\hline
\end{tabular}

\section{PLANTAE LIGNOSE}

\begin{tabular}{|c|c|c|}
\hline & CE & \\
\hline & & \\
\hline A & 1. & s. c. 1979 , Rossia,Barnaul \\
\hline & ren & \\
\hline A & 2. & s. c. 2008 , Rossia, Archangelsk \\
\hline A & 3. & s. c. 1950 , Rossia, Chabarovsk \\
\hline & e & \\
\hline A & 4. & s. c. 2009 , Germany, Greifswald, HB \\
\hline
\end{tabular}

BETULACEAE S. F. Gray

Betula ermanii Cham.

K $5 . \quad$ s. c. 1985 , Rossia, region Mocquaensis

Betula pendula var. carelica (Merckl.) Hämet-Ahti
A
6.
s. c. 1994 , Finland, Tornio, Arb

Betula pendula f. dalecarlica (L. f.) C. K. Schneid.

A $7 . \quad$ s. c. 1994, Finland, Tornio, Arb

CAPRIFOLIACEAE Juss.

Lonicera caerulea ssp. pallasii (Ledeb.) Browicz

A * $8 . \quad$ s. c. 1992, Rossia, Petrozavodsk, HBU

Lonicera chamissoi Bunge ex P. Kir.

A 9. R. 11998 ab s. n. 1982, Rossia, Kamtschatka 
Lonicera chrysantha Turcz. ex Ledeb.

A $10 . \quad$ t. 1950 ab s. c. 1941, Rossia, Archangelsk, Arb

Lonicera edulis Turcz. ex Freyn

A $11 . \quad$ p. c. 2005 ab R. 31962 ab s. c. 1941, Rossia, Samara, HBU

Lonicera ferdinandii Franch.

A $\quad$ * $12 . \quad$ t. c. 1999 ab t. c.1940, Kirghizia, Bischkek

Lonicera involucrata (Richardson) Banks ex Spreng.

$\begin{array}{lll}\text { A } & \text { R. } 22004 \text { ab s. c. 1941, Rossia, Sankt-Peterburg, HBU }\end{array}$

Lonicera nigra L.

A $14 . \quad$ s.n. 1979 ab Ukraine, Carpates

Lonicera orientalis Lam.

A * $15 . \quad$ s. c. 1992 , Rossia, Petrozavodsk, HBU

Lonicera ruprechtiana Regel

A $16 . \quad$ s. c. 1998 , Rossia, Yoshkar-Ola, HB

Lonicera stenantha Pojark.

A $17 . \quad$ s. c. 2000 , Rossia, Barnaul, HBU

Lonicera tatarica L. var. morrowii (A. Gray) Q. E. Yang, Landrein, Borosova \& J. Osborne

\begin{tabular}{lcl}
\hline $\mathrm{A}$ & $* 18$. & s. C. 1992, Rossia, Petrozavodsk, HBU \\
\hline Lonicera xylosteum L. \\
\hline $\mathrm{A}$ & 19. & s. c. 1955, Rossia, Archangelsk, Ar \\
\hline $\mathrm{A}$ & 20. & R. 12001 ab s. c. 1955, Rossia, Archangelsk, Ar \\
\hline
\end{tabular}

CORNACEAE Dumort.

Cornus alba L.

A $21 . \quad$ s. n. 1989, Rossia, Jakutsk

ELAEAGNACEAE Juss.

Hippophäe rhamnoides $\mathrm{L}$.

A 22. p. c. 1987 , Rossia, Archangelsk, Arb

ERICACEAE Juss.

Rhododendron aureum Georgi

\begin{tabular}{lll} 
A & 23. & s. n. 1959, Rossia, Czukotka \\
\hline A & 24. & p. c. 2010 ab s. n. 1959 , Rossia, Czukotka
\end{tabular}

Rhododendron fauriei Franch.

$\begin{array}{lll}\text { A } \quad \text { * } 25 . & \text { s. c. } 1987 \text {, Ukraina, Kiev, HB }\end{array}$ 


\begin{tabular}{|c|c|c|}
\hline A & 26. & s. c. 1988 , Norway, Bergen, HB \\
\hline \multicolumn{3}{|c|}{ Rhododendron caucasicum Pall. } \\
\hline A & 27. & R.1 1954 abs p. n. 1937, Georgia, Bakuriani \\
\hline A & 28. & p. n. 1955, Georgia, Bakuriani \\
\hline \multicolumn{3}{|c|}{ Rhododendron myrtifolium Schott et Kotschy } \\
\hline A & 29. & s. n. 1956, Ukraine, Carpates \\
\hline \multicolumn{3}{|c|}{ FABACEAE Lindl. } \\
\hline \multicolumn{3}{|c|}{ Caragana arborescens Lam. } \\
\hline A & 30. & R. 11974 ab s. c. 1952, Rossia, Tomsk, HB \\
\hline A & 31. & R. 22010 ab s. c. 1952 , Rossia, Tomsk, HB \\
\hline A & 32. & R. 12010 ab s. c. 1989, Finland, Tornio, Arb \\
\hline \multicolumn{3}{|c|}{ Caragana boissii C. K.Schneid. } \\
\hline A & 33. & R. 11963 ab s. c. 1956, Rossia, Archangelsk, Arb \\
\hline \multicolumn{3}{|c|}{ Caragana grandiflora (M. Bieb.) DC. } \\
\hline $\mathrm{K}$ & 34. & s. c. 1990 , Rossia, Archangelsk, Ar \\
\hline
\end{tabular}

\section{GROSSULARIACEAE DC.}

Ribes alpinum $\mathrm{L}$.

A $35 . \quad$ s. n. 1983, Suisse, Chambesy / Geneve, HB

Ribes hudsonianum Richardson

A $36 . \quad$ s. c. 1991, Rossia, Zagorsk, region Mosquaensis

Ribes komarovii Pojark.

A * $37 . \quad$ s. c. 1984 , Ukraine, Kharkov, HBU

Ribes nigrum L. var. sibiricum W. Wolf. |

A $38 . \quad$ t. 2010 abs s. c. 1954, Rossia, Barnaul

Ribes niveum Lindl.

A $39 . \quad$ t. 2010 ab s. c. 1998, Germany, Leipzig, HBU

Ribes rubrum L.
A
40.
s. c. 1997 , Rossia, Sankt-Peterburg, HBIB

\section{PINACEAE Lindl.}

Picea mariana (Mill.) Britton, Sterns \& Poggenb.

K $41 . \quad$ s. c. 1990 , Finland, Rovaniemi

RANUNCULACEAE JUSS.

Clematis alpina ssp. ochotensis (Pall.) Kuntze 


\begin{tabular}{lcl}
\hline A $* 42$. & s. n. 1989, Rossia, Jakutsk \\
\hline \multicolumn{2}{l}{ Clematis integrifolia L. } \\
\hline A 43. & s. c. 2008 , Bjelorossia, Minsk, HBU \\
\hline Clematis flammula L. & \\
\hline A & 44. & s. c. 2011 , Romania, Cluj, HBU \\
\hline
\end{tabular}

ROSACAEA Juss.

Amelanchier spicata (Lam.) K. Koch

\begin{tabular}{llll}
\hline$A$ & $*$ & 45. & s. c. 1955, Rossia, Ekaterinburg, HB \\
\hline$A$ & $*$ & 46. & R. 12010 ab s. c. 1955, Rossia, Ekaterinburg, HB \\
\hline A & $*$ & 47. & t. c. 2010 ab s. c. 1955, Rossia, Ekaterinburg, HB
\end{tabular}

Cotoneaster alaunicus Golitsin

K $48 . \quad$ s. c. 1957 , Rossia, Voroneg, HBU

Cotoneaster integerrimus Medik.

A $49 . \quad$ p. c. 1989 , Finland, Tornio, Arb

Crataegus dahurica Koehne ex C. K. Schneid.

A $50 . \quad$ s. n. 1979, region Khabarovskensis

Crataegus flabellata (Bosc ex Spach) K. Koch

A $51 . \quad$ s. c. 1998 , Rossia, Sankt-Peterburg, HBIB

Crataegus maximowiczii C. K. Schneid.

A $52 . \quad$ s. c. 1998, Rossia, Archangelsk, Arb

Crataegus pentagyna Waldst.et Kit.

A * $53 . \quad$ s. c. 1998 , Rossia, Archangelsk, Arb

Crataegus pinnatifida Bunge

A $54 . \quad$ s. c. 1997, Rossia, Archangelsk, Arb

Crataegus $\times$ schroederii (Regel) Kochne

A * $55 . \quad$ s. c. 1990 , Rossia, Archangelsk, Arb

Prunus asiatica Kom.

A $56 . \quad$ s. n. 1989, Rossia, Jakutia

Prunus padus L. fil. colorata Almquist

A $57 . \quad$ t. 2009 ab p. c. 1989, Finland, Tornio, Arb

Rosa acicularis Lindl.

\begin{tabular}{lll}
\hline A & 58. & s. c. 1989, Finland, Tornio, Arb \\
\hline A & 59. & s. n. 1995, Rossia, Karelia \\
\hline
\end{tabular}

Rosa gallica L.

A $60 . \quad$ s. c. 1998, Germany, Erlangen, HB

Rosa glauca Pourr. 


\begin{tabular}{lll}
\hline A & 61. & s. c. 1957, Netherland, Leiden, HB \\
\hline A & 62. & R. 22009 ab s. c. 1957, Netherland, Leiden, HB \\
\hline A & 63. & R. 11988 ab s. n. 1980, Rossia, Karelia, Sortavala \\
\hline Rosa laxa Retz. & \\
\hline A & 64. & t. 2010 ab t. 1951, Rossia, Kirovsk, HB \\
\hline Rosa rugosa Thunb. & \\
\hline A & 65. & s. n. 1982, Rossia, Kamtschatka \\
\hline A & 66. & s. c. 2009, Rossia, Barnaul
\end{tabular}

Sorbus sambucifolia (Cham. \& Schltdl.) M. Roem.

\begin{tabular}{lll}
\hline A & 67. & R. 21979 ab. s. n. 1947, Rossia, Oriens eretremus \\
\hline A & 68. & s.n. 1984, Rossia, Kamtschatka
\end{tabular}

Spiraea media Schmidt

A $69 . \quad$ s. c. 2010 , Rossia, Tver, HBU

SAMBUCACEAE Batsch ex Borkh.

Sambucus racemosa $\mathrm{L}$.

A 70 s. c. 2013, Rossia, Syktyvkar, HB

SOLANACEAE Juss.

Solanum dulcamara L.

A $71 . \quad$ s. c. 2013 , Rossia, Syktyvkar, HB

VIBURNACEAE Rafin. |

Viburnum opulus L.

A $72 . \quad$ s. c. 1980 , Rossia, Vaalam, Karelia

\section{PLANTAE HERBACEAE}

ALLIACEAE J. Agardh

Allium atrosanguineum Kar.et Kir.

73. R. 21959 ab p. n. 1937, Kazachstan, Alatau

74. s. n. 1986, Kazachstan, Alatau

Allium beesianum W. W. Sm.

\begin{tabular}{lll}
\hline A & 75. & p. n. 2006, Rossia, Mosqua
\end{tabular}

Allium cernuum Roth

A $76 . \quad$ s. C. 1998 , Italia, Bormio, HB

Allium fedtschenkoanum Regel

77. s. n. 1990, Tadschikistan, Chorog, HB Pamirensis

Allium fistulosum $\mathrm{L}$.

A 78 s. c. 2015 , Rossia, Izhevsk, HBU 


\section{Allium gultschense B. Fedsch. |}

\begin{tabular}{lll}
\hline A & 79. & s. c. 2000 , Rossia, Samara, HBU
\end{tabular}

Allium ledebourianum Schult. et Schult. fil.
A
80.
s. n. 1990, Tadschikistan, Chorog, HB Pamirensis

Allium microdictyon Prokh.
A
81.
s. n. 2000 , Rossia, Barnaul, HBU

Allium oliganthum Kar. et Kir.
A
82.
p. n. 2006, Rossia, Mosqua, HBP

Allium pskemense B. Fedtsch.
A
83.
s. c. 1988 , Bjelorossia, Minsk, HBU

Allium schoenoprasum $\mathrm{L}$.

\begin{tabular}{cl}
\hline 84. & p. n. 1972, Rossia, Altai, 1 \\
\hline 85. & R. 11983 ab p. n. 1972, Rossia, Altai, 1 \\
\hline 86. & p. n. 1974, Ukraine, Carpates \\
\hline 87. & s. n. 1981, Canada, Montreal, HB \\
\hline 88. & p. n. 1974, Ukraine, Carpates \\
\hline Allium schoenoprasum L. ssp. alpinum (DC.) Čelak.
\end{tabular}

89. s. n. 1985, Czechia, Liberec, HB

Allium victorialis $\mathrm{L}$.

90. p. n. 1999 , ab p. n. 1934 , Rossia, Altai, 2

AMARYLLIDACEAE J. St.-Hil. |

Narcissus angustifolius Curt.

$91 . \quad$ p. n. 1988 , Ukraine, Carpates

APIACEAE Lindl.

Angelica genuflexa Nutt. ex Torr. et Gray

92. s. n. 1983, Rossia, Kamtschatka

Anthriscus nitida (Wahlenb.) Hazslinszky

93. s. c. 1980 , Ukraine, Carpates

Astrantia major L.

94. s. n. 1997, Austria Graz, HBU

Astrantia trifida Hoffm

95. S. c. 1995 , Rossia, Mosqua, HBP

Chaerophyllum villarsii Koch.

96. S. n. 1991, Austria, Salzburg, HBU

Heracleum antasiaticum Manden. 
97. s. c. 1954 , Armenia, Erevan, HBIB

Heracleum calcareum Albov var. colchicum (Lipsky) Satzyperova

$98 . \quad$ s. n. 1955, Rossia, Mosqua, HBP

Heracleum cyclocarpum C. Koch

$99 . \quad$ s. n. 1954, Georgia, Tbilici, HB

Heracleum lanatum Michx.

$100 . \quad$ s. n. 1956, Canada, Toronto, HBU

Heracleum lehmannianum Bunge

101. s. n. 1949, Rossia, Sankt-Peterburg, HBIB

Heracleum platytaenium Boiss.

102. s. c. 1957 , Polania, Warszawa, HBU

Heracleum sibiricum $\mathrm{L}$.

103. s. n. 1957 , France, Paris, HB

Heracleum sphondilium L. ssp. pyrenaicum (Lam.) Bonnier \& Layens

104. s. n. 1994, Austria, Salzburg, HBU

Laserpitium latifolium $\mathrm{L}$.

105. p. n. 1956, Ukraine, Carpates, 2

Laserpitium siler L.

A $106 . \quad$ s. c. 2007 , Italia, Aosta, HB

Ligusticum mutellina (L.) Crantz

107. s. n. 1980 , Ukraine, Carpates

Myrrhis odorata (L.) Scop.

$108 . \quad$ s. c. 1956, Germany, Rostok, HBU

109. R. 11975 ab s. c. 1956, Germany, Rostok, HBU

ASPHODELACEAE R. Br.

Paradisea liliastrum (L.) Bertol.

110. S. c. 1946 , Rossia, Nizhny Novgorod, HBU

ASTERACEAE Dumort.

Achillea impatiens L.

111. R. 11989 ab c. n. 1947, Rossia, Igarga

Adenostyles platyphylloides (Somm. et Levier) Czer.

112. s. n. 1976, Caucasus

Antennaria howellii Greene

113. s. c. 2003, Hungary, Vácrátót, HB

Arnica angustifolia Vahl. 
114. S. c. 1957 , Sweden, Stockholm, HBU

Arnica angustifolia Vahl. ssp. alpina (L.) I. K. Ferguson

115. R. 21970 ab s. c. 1965, Sweden, Uppsala, HBU

Arnica chamissonis Less. ssp. genuine Maguire

$116 . \quad$ s. c. 1981 , Canada, Vancouver, HBU

$117 . \quad$ s. c. 1983 , Suisse, Champex-Las HB

Arnica cordifolia Hook.

118. S. c. 2003 , France, Nancy, HBU

$119 . \quad$ s. c. 2003 , France, Nancy, HBU

Arnica griscomii Fernald ssp. frigida (C. A. Mey. ex Iljin) S. J. Wolf

$120 . \quad$ s. c. 1997 , Iceland, Akureyri, HB

Arnica lanceolata Nutt. ssp. prima (Maguire) Strother \& S. J. Wolf

\begin{tabular}{cc}
\hline A 121. & s. c. 2002, Germany, München, HB \\
\hline Arnica longifolia Eaton & \\
\hline 122. & s. c. 1984, Hungary, Vácrátót, HB \\
\hline
\end{tabular}

Arnica mollis Hook.

\begin{aligned} & \hline 123. s. c. 1972 , Danmark, København, HBU \\ & \hline 124. s. c. 1996, Germany, Marburg, HBU \\ & \hline\end{aligned}

Arnica montana L.

\begin{tabular}{lll}
\hline A & 125. & R. 41951 ab p. n. 1933, Rossia, Sankt-Peterburg, HBIB \\
\hline & 126. & s. n. 1999, Sweden, Lund, HB
\end{tabular}

Arnica nevadensis A. Gray

$127 . \quad$ s. c. 1970 , Germany, Gießen, HBU

$128 . \quad$ s. c.1992, Rossia, Syktyvkar, HB

Arnica parryi A. Gray

\begin{tabular}{|c|c|}
\hline 129. & s. c. 1969 , Suisse, Champex-Lac, HB \\
\hline 130. & s. c. 1997, Iceland, Akureyri, HB \\
\hline 131. & s. c. 1998 , Iceland, Reykjavik, HB \\
\hline \multicolumn{2}{|c|}{ Arnica rydbergii Greene } \\
\hline 132. & s. c. 1983, Canada, Edmonton, HBU \\
\hline \multicolumn{2}{|c|}{ Arnica sachalinensis (Regel) A. Gray } \\
\hline 133. & p. n. 1996, Rossia, Sachalin \\
\hline \multicolumn{2}{|l|}{ Arnica sororia Greene } \\
\hline \multirow[t]{2}{*}{$A$} & s. c. 1992, Germany, Bonn, HBU \\
\hline & s. c. 1993 , Netherland,Rotterdam, HB \\
\hline \multicolumn{2}{|c|}{ Artemisia arctica (Besser) Leonova } \\
\hline 136. & R. 1 1970, ab s. n. 1959, Rossia, Czukotka, 3 \\
\hline
\end{tabular}




\begin{tabular}{|c|c|}
\hline \multicolumn{2}{|l|}{ Aster sibiricus L. } \\
\hline 137. & s. c. 1997 , Latvia, Salaspis, HB \\
\hline \multicolumn{2}{|c|}{ Bellis caerulescens (Coss.) Coss. et Bol. } \\
\hline 138. & R. 1 1989, ab s. c.1956, France, Paris, HB \\
\hline \multicolumn{2}{|c|}{ Centaurea nervosa Willd. } \\
\hline 139. & s. n. 1999, Suisse, Chambesy / Geneve, HB \\
\hline \multicolumn{2}{|c|}{ Cyanus montana (L.) Hill. cv. Alba } \\
\hline 140. & R. 1 1980, ab s. c.1967, France, Besanson, HBU \\
\hline \multicolumn{2}{|c|}{ Lactuca alpina (L.) A. Gray } \\
\hline 141. & s. c. 1957 , Slovakia, Bratislava, HBU \\
\hline \multicolumn{2}{|c|}{ Lactuca plumieri (L.) Gren. \& Godr. } \\
\hline 142. & R. 1 1953, ab s. n. 1938, Danmark, København, HBU \\
\hline \multicolumn{2}{|c|}{ Cirsium spinosissimum (L.) Scop. } \\
\hline 143. & s. n. 1999, Suisse, Chambesy / Geneve, HB \\
\hline \multicolumn{2}{|c|}{ Doronicum altaicum Pall. } \\
\hline 144. & s. n. 1969, Kazachstan, Leninogorsk, HB \\
\hline
\end{tabular}

145. S. n. 1991,Sweden, Uppsala, HBU

Doronicum cataractarum Widder

146. R. 2 1959, ab s. n. 1938, Austria, Graz, HBU

Doronicum clusii (All.) Tausch

147. s. n. 1982, Suisse, Lausanne, HB

Doronicum glaciale (Wulf.) Nyman

148. S. c. 2014 , Iceland, Akureyri, HB

Doronicum grandiflorum Lam.

149. R. 3 1980, ab s. n. 1939, France, Grenoble, HB Alp.

150. S. n. 1998, Suisse, Chambésy / Genéve, HB

Doronicum macrophyllum Fisch. ex Hornem.

151. s. n. 1956, Armenia, Erevan, HB

Doronicum oblongifolium DC.

152. R. 1 1949. ab s. n. 1938, Armenia, Erevan, HB

153. R. 1 1949. ab s. n. 1938, Armenia, Erevan, HB

Doronicum turkestanicum Cavill.

154. s. n. 1986, Kazachstan, Alatau

Erigeron multiradiatus (Lindl. ex DC.) Benth. et Hook.

155. R. 4 1976, ab s. c. 1939 , Finland, Helsinki, HBU

Erigeron peregrinus (Banks ex Pursh) Greene ssp. callianthemus (Greene) Cronq. 
156. s. c. 1989 , Iceland, Akureyri, HB

\begin{tabular}{|c|c|c|}
\hline \multicolumn{3}{|c|}{ Hieracium sabaudum L. } \\
\hline A & 157. & s. c. 2015 , Germany, Stuttgart, HB \\
\hline \multicolumn{3}{|c|}{ Homogyne alpina (L.) Cass. } \\
\hline & 158. & p. n. 1979 , Ukraine, Carpates, 2 \\
\hline \multicolumn{3}{|c|}{ Jacobaea subalpina (W. D. J. Koch) Pelser \& Veldkamp } \\
\hline & 159. & s. c. 2009 , Germany, Berlin-Dahler \\
\hline
\end{tabular}

Ligularia alticola Vorosch.

160. S. n. 1994, Rossia, Sachalin

Ligularia calthifolia Maxim.

161. R. 1 1975, ab s. n. 1947, Rossia, Oriens eretremus

Ligularia thomsonii Pojark.

162. s. n. 1953, Rossia, Sankt-Petersburg, HBIB

Prenanthes purpurea $\mathrm{L}$.

163. s. n. 1986, France, Nancy, HBU

\section{Senecio nemorensis L.}

164. s. n. 1972, Finland, Helsinki, HBU

Senecio nemorensis L. ssp. jacquinianus Čelak

165. S. n. 1988, Czechia, Liberec, HB

166. R. 11964 ab s. n. 1957, Slovakia, Bratislava, HBU

Stemmacantha carthamoides (Willd.) M. Dittrich

167. p. n. 1987, Rossia, Altai

Tanacetum vulgare $\mathrm{L}$.

168. R. 2 1998, ab c. n. 1947, Rossia, Igarga

Trommsdorfia uniflora (Vill.) Soják

169. S. c. 1956 , Rossia, Mosqua, HBP

\section{BERBERIDACEAE Juss.}

Sinopodophyllum hexandrum (Royle) T. S. Ying

\begin{tabular}{lll}
\hline A & 170. & s. c. 1995, China \\
\hline A & 171. & s. c. 2006, Rossia, Sankt-Peterburg, HBIB \\
\hline A & 172. & s. c. 2013 , Iceland, Reykjavik, HB
\end{tabular}

BIGNONIACEAE Juss.

Incarvillea zhongdianensis C. Gray-Wilson

A $173 . \quad$ s. c. 2000 , lceland, Reykjavik, HB 


\begin{tabular}{|c|c|}
\hline \multicolumn{2}{|c|}{ Adenophora lilifolia (L.) A. DC. } \\
\hline 174. & R. 1 1954, ab s. n. 1947, Kazachstan \\
\hline \multicolumn{2}{|c|}{ Campanula barbata L. } \\
\hline 175. & R. 4 1981, ab s. n. 1939, France, Samoëns, HB \\
\hline \multicolumn{2}{|c|}{ Campanula bayerniana Rupr. } \\
\hline 176. & s. c. 1983 , Germany, Kiel, HBU \\
\hline \multicolumn{2}{|c|}{ Campanula glomerata L. } \\
\hline 177. & p. c. 1998 , ab R.1 1975, ab s. n. 1964 , Ukraine, Carpates \\
\hline 178. & p. c. 1998, ab s. c. 1941 , Canada, Ottava, HB \\
\hline \multicolumn{2}{|c|}{ Campanula glomerata L. var. acaulis hort. } \\
\hline 179. & p. c. 1998 , ab s. c. 1946 , Rossia, Sankt-Peterburg, HBIB \\
\hline \multicolumn{2}{|c|}{ Campanula glomerata L. ssp. caucasica (Trautv.) Ogan. } \\
\hline 180. & s. n. 1984, Caucasus \\
\hline \multicolumn{2}{|l|}{ Campanula latifolia L. } \\
\hline 181. & s. c. 1976, Rossia, Ekaterinburg, HBU \\
\hline 182. & p. c. 1995 , ab s. c. 1939 , Rossia, Sankt-Peterburg, HBIB \\
\hline 183. & s. c. 2000 , Rossia, Petrosavodsk, HBU \\
\hline
\end{tabular}

184. R. 2 1959, ab p. n. 1939, Georgia, Bakuriani

Phyteuma betonicifolium Vill.

A $185 . \quad$ s. n. 2007, Austria, Salzburg, HBU

Phyteuma orbiculare L.

186. s. n. 1976, Austria, Wien, HBU

Phyteuma spicatum L.

187. S. n. 1999, Suisse, Chambésy / Genéve, HB

Phyteuma vagneri A. Kerner

$188 . \quad$ s. n. 1980 , Ukraine, Carpates

\section{CARYOPHYLLACEAE Juss.}

Dianthus alpinus L.

A $189 . \quad$ s. n. 1993, Austria, Wien, HBU

Dianthus barbatus L. var. compactus (Kit.) Heuff.

A $190 . \quad$ s. c. 2017 , Germany, Rabenau

Dianthus eretmopetalus Stapf

A $191 . \quad$ s. c. 2004, Ireland, Dublin, HB

Dianthus deltoides L. cv. Maiden Pink 


A $192 . \quad$ s. c. 2004 , Slovakia, Bratislava, HBU

Dianthus gratianopolitanus Vill. cv. Purpur Konig

$\begin{array}{lll}\text { A } & 193 . & \text { s. c. 2004, Romania, Cluj, HBU }\end{array}$

Dianthus japonicus Thunb.

A $194 . \quad$ s. c. 2004, Italia, Courmayeur, HB

Dianthus orientalisAdams

A $195 . \quad$ s. c. 1990 , Norway, Bergen, HBU

Dianthus superbus L.

A $196 . \quad$ s. n. 1998, Italy, Bormio, H Alp.

Dianths webbianus Parl. ex Vis.

A $197 . \quad$ s. c. 2017, Rossia, Blagoveschensk, HB

Silene dioica (L.) Clairv.

198. s. n. 1989 , Romania

Lychnis haageana Bailly

A 199 s. c. 2001 , Polonia, Cracovia, HBU

Saponaria lutea $\mathrm{L}$.

A $200 . \quad$ s. c. 1998 , Germany, Halle, HBU

Silene flos-jovis (L.) Greuter \& Burdet

A $201 . \quad$ s. c. 2014 , Rossia, Kirov, HBU

Silene viscaria (L.) Jess. cv. Splendens

A 202. S. c. 2004 , Clovakia, Bratislava, HBU

Silene vulgaris (Moench) Garcke

203. S. n. 1972 Rossia, Narjan-Mar, flora localis

CONVALLARIACEAE Horan.

Convallaria keiskei Miq. cv. Albostriata

$204 . \quad$ p. n. 2009, Rossia, Orel

Streptopus streptopoides (Ledeb.) Frye et Rigg

$205 . \quad$ p. n. 1967, Rossia, Sachalin

CORNACEAE Dumort.

Cornus canadensis L.

206. s. n. 1956 , Canada, Ottawa, HB

\section{CRASSULACEAE DC.}

Rhodiola heterodanta (Hook. fil. et Thoms.) Boriss.

207. S. n. 1991, Austria, Salzbrug, HBU 


\begin{tabular}{|c|c|c|}
\hline \multicolumn{3}{|c|}{ Rhodiola integrifolia Rafin. } \\
\hline & 208. & R. 1 1967, ab s. n. 1959, Rossia, Czukotka, 2 \\
\hline & 209. & p. n. 1982, Rossia, Kamtschatka \\
\hline A & 210. & p. n. 1987, Rossia, Kamtschatka \\
\hline \multicolumn{3}{|c|}{ Rhodiola ishidae (Miyabe et Kudo) Hara } \\
\hline$A$ & 211. & s. c. 2001 , Iceland, Reykjavik, HB \\
\hline \multicolumn{3}{|c|}{ Rhodiola stephanii (Cham.) Trautv. et C. A. Mey. } \\
\hline & 212. & s. c. 1957, Rossia, Sankt-Petersburg, HBIB \\
\hline \multicolumn{3}{|c|}{ Sedum roseum (L.) Scop. } \\
\hline & 213. & R. 1 1972, ab s. n. 1959, Rossia, Czukotka, 4 \\
\hline & 214. & R. 1 1972, ab s. n. 1957, Ukraine, Carpates 1 \\
\hline & 215. & p. n 1998, ab s. n. 1957, Ukraine, Carpates 1 \\
\hline & 216. & s. n. 1981, Rossia, Polar Ural \\
\hline
\end{tabular}

\section{FABACEAE Lindl.}

Hedysarum alpinum L.
217.
p. n. 1989, Rossia, region Murmanensis

Lathyrus frolovii Rupr.

218. R. 2 1987, ab s.c.1947, Ireland, Dublin, HB

Lathyrus gmelinii Fritsch

$\begin{array}{ll}219 . & \text { s. c. } 1954 \text {, Rossia, Tomsk, HBU } \\ 220 . & \text { s. c. } 1954, \text { Rossia, Mosqua, HBP }\end{array}$

Thermopsis rhombifolia (Pursh) Richardson

A 221 s. c. 1998, Belquim, Meise, HB

\section{GENTIANACEAE Juss.}

Gentiana dschungarica Regel

\begin{tabular}{cl}
\hline 222. & p. n. 1969, Rossia, Altai, 1 \\
\hline 223. & R. 1 1990, ab p. n. 1969, Rossia, Altai, 1 \\
\hline Gentiana lutea L. & \\
\hline 224. & s. c. 1957, France, Grenoble HB Alp. \\
\hline 225. & s. n. 1980, Suisse, Chambésy / Genéve, HB \\
\hline 226. & s. n. 1980, Suisse, Chambésy / Genéve, HB \\
\hline 227. & s. n. 1981, France, Besancon, HBU \\
\hline 228. & s. n. 1981, Sweden, Zürich, HBU \\
\hline 229. & s. n. 1982, Italia, Genova, HBI
\end{tabular}

Gentiana septemfida Pall. 


\section{R. 2 1990, ab 1947, Georgia, Bakuriani}

\section{GERANIACEAE Juss.}

Geranium clarkei P. Y | Yeo cv. Kashmir Purple

$$
231 . \quad \text { s. c. } 2004 \text {, Iceland, Akureyri, HB }
$$

Geranium columbinum L.

$$
\text { 232. S. c. } 2013 \text {, Suisse, Sankt-Gallen, HB }
$$

\section{Geranium himalayense Klotzsch}

A $233 . \quad$ s. n. 1985 , Kirghizia

Geranium macrorrhizum L.

A 234 s. c. 2002, Italia, Bormio, HAlp

Geranium transbaicalicum Serg.

$235 . \quad$ s. c. 1958 , Rossia, Jakutsk

\section{HEMEROCALLIDACEAE R.Br.}

Hemerocallis dumortieri Morr.
236.
s. n. 1947, Rossia, Yuzhno-Sakhalinsk, HB

Hemerocallis esculenta Koidz.

237. s. n. 1967, Rossia, Yuzhno-Sakhalinsk, HB

\section{HYACINTHACEAE Batsch}

Ornithogalum balansae Boiss.

238. R. 2 1954, ab p. n. 1946, Georgia, Bakuriani

HYPERICACEAE Juss.

Hypericum ascyron L. ssp. gebleri (Ledeb.) N. Robson.

A 239 . s. c. 2014, Rossia, Ekaterenburg, HB

Hypericum barbatum Jacq.

A $240 . \quad$ s. c. 2013 , Suisse, Sankt-Gallen, HB

Hypericum perforatum $\mathrm{L}$.
A
241.
s. c. 1992, England, Surrey, HB

IRIDACEAE Juss.

Iris setosa Pall. ex Link

242. S. n. 1959, Rossia, Jakutsk, 2

243. R. 1 1964, ab s. n. 1959, Rossia, Jakutsk, 2

244. s. n. 1971 , Rossia, Jakutsk, 3

$245 . \quad$ p. n. 1982, Rossia, Kamtschatka 


\section{Iris sibirica L.}

A 246. $\quad$ p. n. 2002, Rossia, Irkutsk

\section{LAMIACEAE Lindl.}

Nepeta nuda L.

$\begin{array}{lll}\text { A } & 247 . & \text { s. c. } 2008 \text {, Italia, Trento, } \mathrm{HB}\end{array}$

Nepeta schugnanica Lipsky

$248 . \quad$ s. c. 1996 , Iceland, Akureyri, HB

Stachys alopecuros Benth.

249. s. n. 1995, Austria, Graz, HBU

Stachys macrantha (C. Koch) Stearn

\begin{tabular}{ll}
\hline 250. & p.n. 1971, Georgia, Bakuriani \\
\hline 251. & R.1 1974, ab p.n. 1939, Georgia, Bakuriani \\
\hline 252. & R.1 1975, ab p.n. 1939, Caucasus, Tschinvali \\
\hline
\end{tabular}

\section{LILIACEAE Juss.}

Erythronium sibiricum ( Fisch. et C. A. Mey.) Kryl.

253. p. c. 2006 , ab R. 1 1949, ab p. n. 1936, Rossia, Sajany

Fritillaria camschatcensis (L.) Ker-Gawl.

254. p. n. 1982, Rossia, Kamtschatka

\section{Fritillaria latifolia Willd.}

$255 . \quad$ p. n. 1973 , Caucasus

Lilium martagon L.

\begin{tabular}{ll}
\hline 256. & s. n. 1980, Ukraine, Carpates \\
\hline 257. & p. n. 1986, Ukraine, Carpates \\
\hline 258. & p. c. 2005, ab p. n. 1934, Rossia, Altai, 1 \\
\hline
\end{tabular}

LINACEAE DC. ex S. F. Gray

\section{Linum perenne L.}

A * 259. $\quad$ s. c. 2002, Suisse, Porrentruy, HB

MALVACEAE Juss.

Malva moschata L.

260. S. c. 2014 , Lithuania, Vilnius, HB

\section{PAEONIACEAE Rudolphi}

Paeonia anomala L.

\begin{tabular}{ccc}
\hline & 261. & R. 11951 ab p. n. 1934, Rossia, Altai, 4 \\
\hline A & p. c. 2008 ab R. 21976 ab p. n. 1934, Rossia, Altai, 4 \\
\hline
\end{tabular}




\begin{tabular}{|c|c|}
\hline 263. & p. c. 2006 ab R. 21976 ab p. n. 1934, Rossia, Altai, 4 \\
\hline 264. & p. c. 1974 , Rossia, Archangelsk, Arb \\
\hline \multicolumn{2}{|c|}{ Paeonia lactiflora Pall. } \\
\hline 265. & s. c. 2006 , Ukraine, Kiev, HB \\
\hline \multicolumn{2}{|c|}{ Paeonia mascula (L.) Mill. } \\
\hline 266. & s.c. 2005 , France, Caen, HB \\
\hline \multicolumn{2}{|l|}{ Paeonia officinalis L. } \\
\hline 267. & s.n. 1979, Germany, Berlin, HB \\
\hline \multicolumn{2}{|c|}{ Paeonia oreogetonS. Moore } \\
\hline 268. & s. c. 1954 , Rossia, Mosqua, AAT \\
\hline \multicolumn{2}{|c|}{ PAPAVERACEAE Juss. } \\
\hline \multicolumn{2}{|l|}{ Chelidonium majus L. } \\
\hline 269. & s. c. 2012 , Germany \\
\hline \multicolumn{2}{|c|}{ Papaver cambricum L. } \\
\hline 270. & s. c. 2015 , Germany \\
\hline
\end{tabular}

Papaver nudicaule L.

271. R ... ab s.c. 1938, Finland, Helsinki, HBU

Papaver oreophilum Rupr.

272. p. n. 1955, Caucasus, Tschinvali

Papaver orientale L.

273. R ... ab s. c. 1945 , Rossia, Perm, HBU

Papaver orientale L. cv. Pizzicato

A 274 s. c. 2000 , Rossia, Yoshkar-Ola, HBI

Papaver orientale L. cv. Scharlachkonig

A $275 . \quad$ s. c. 2004 , lceland, Akureyri, HB

Papaver orientale L. cV. Blutrot

A 276 s. c. 2004 , Iceland, Akureyri, HB

Papaver orientale L. cv. Haides Blush

A 277 s. c. 2015 , Germany, Essen, HB

PLANTAGINACEAE Juss.

Plantago atrata Hoppe

278. s. n. 1999, Austria, Salzburg, HBU

POACEAE Barnhart

Elymus mutabilis (Drob.) Tzvel. 


\begin{tabular}{|c|c|}
\hline 279. & R. 1 1990, ab s. n. 1947, Rossia, Igarka \\
\hline \multicolumn{2}{|l|}{ Phleum alpinum L. } \\
\hline 280. & s. n. 1951, France,GrenobleHB Alp. \\
\hline \multicolumn{2}{|c|}{ POLEMONIACEAE Juss. } \\
\hline \multicolumn{2}{|c|}{ Polemonium brandegeei (A. Gray) Greene } \\
\hline 281. & s. c. 2002, Hungary, Vácrátòt, HB \\
\hline \multicolumn{2}{|c|}{ POLYGONACEAE Juss. } \\
\hline \multicolumn{2}{|c|}{ Persicaria alpina (All.) H. Gross } \\
\hline 282. & s. c. 1984 , Rossia, Sankt-Petersburg, HBIB \\
\hline \multicolumn{2}{|c|}{ Persicaria bistorta (L.) Samp. } \\
\hline 283. & p. n. 1953, Rossia, Naltchik, HBU \\
\hline 284. & p. n. 1971, Georgia, Bakuriani \\
\hline 285. & p. n. 1969, Rossia, Altai \\
\hline 286. & p. n. 1979, Ukraine, Carpates \\
\hline
\end{tabular}

Polygonum tripterocarpum A. Grey ex Rothr. |

$287 . \quad$ s. n. 1959 , Rossia, Jakutsk, 3

Polygonum weyrichii F. Schmidt

288. S. c. 1951 , Ukraine, Dniepropetrovsk, HBU

Rheum alexandrae Batal.

$289 . \quad$ s. c. 1958 , Finland, Helsinki, HBU

Rheum compactum L.

$290 . \quad$ s. c. 1980 , Rossia, regio Khabarovskensis

Rheum crassinervium Fisch. ex Lindl.

291. S. c. 1999 , Romania, Cluj, HBU

Rheum kialense Franch.

292. s. c. 1958 , Sweden, Uppsala, HBU

Rheum macrocarpum Losinsk.

293. S. c. 1957 , Rossia, Sankt-Petersburg, HBIB

Rheum ribes $\mathrm{L}$.

\begin{tabular}{ll}
\hline 294. & s. c. 1950, Ukraine, Dniepropetrovsk, HBU \\
\hline 295. & s. c. 1984, Rossia, Mosqua, NPO VILAR \\
\hline
\end{tabular}

Rheum spiciforme Royle

\begin{tabular}{lll}
\hline & 296. & s. c. 1946, Rossia, Mosqua, AAT \\
\hline A & 297. & s. c. 2001 , Italia, Bormio, H Alp
\end{tabular}

Rheum tataricum L. fil. 


\begin{tabular}{|c|c|c|}
\hline & 298. & R. 2002 , ab s. c. 1939 , Rossia, Mosqua, AAT \\
\hline \multicolumn{3}{|c|}{ Rheum tetragonopus L. } \\
\hline & 299. & s. c. 1949 , Rossia, Mosqua, NPO \\
\hline \multicolumn{3}{|c|}{ Rheum webbianum Royle } \\
\hline & 300. & s. c. 1947 , Rossia, Mosqua, AAT \\
\hline$A$ & 301. & s. c. 1999 , Germany, Leipzig, HBU \\
\hline
\end{tabular}

\section{PRIMULACEAE Vent.}

Androzace laggeri A. Huet |

302. S. c. 1968, Suisse, Chambésy/Genéve, HB

Androzace montana (A. Gray) Wendelbo

A $303 . \quad$ s. c. 2004 , Iceland, Akureyri, HB

Dodecatheon jeffreyi Moore

304. s. n. 1995, USA, Berkey, HBU

Dodecatheon meadia L.

$305 . \quad$ s. c. 1982 , England, Wisley

Primula amoena M. Bieb.

306. R 2005, ab s. c. 1964, Rossia, Mosqua, HBP

307. | 3. c. 1985, Rossia, Mosqua, HBP

Primula carpathica (Griseb. et Schenk) Fuss

\begin{tabular}{lll}
\hline A & 308. & s. n. 1991, Germany, Oberhof, HB \\
\hline 309. & s. n. 1991, Germany, Oberhof, HB
\end{tabular}

Primula clusiana Tausch

310. s. n. 1985, Austria, Wien, HBU

Primula elatior (L.) Hill

311. S. n. 1986, Austria, Wien, HBU

312. s. n. 1986, Austria, Wien, HBU

Primula elatior(L.) Hill ssp. intricata (Greene. | et Gerd.) Lüdi

313. p. n. 1984, Ukraine, Carpates

Primula elatior (L.) Hill ssp. tatrensis (Domin) Soò

314. s. n. 1995, Slovakia, Bratislava, HBU

315. R. 12008 , ab s. n. 1939, Polonia, Cracovia, HBU

Primula poloninensis (Domin) Fed.

316. R. 2 1975, ab p. n. 1956, Ukraine, Carpates

Primula poloninensis (Domin) Fed. f. violacea

317. R. 1 1975, ab p. n. 1956, Ukraine, Carpates, 2

Primula saguramica Gavl. 
318. R. 2 2002, ab p. n. 1966, Rossia, Naltchik, HBU

Soldanella alpina L.

319. p. n. 2010 ab s. c. 1956 , Suisse, Porrentruy, HB

Soldanella hungarica Simonk.

320. p. n. 1979, Ukraine, Carpates

RANUNCULACEAE Juss.

Aconitum anthoroideum DC.

321. R. 11974 , ab s. c. 1969 , Rossia, Novosibtrsk, HBU

Aconitum barbatum Pers.

322. R. 11980 , ab p. n. 1970, Rossia, Altai

Aconitum $\times$ cammarum L.

323. p. n. 1974, Ukraine, Carpates
324. p. n. 1979, Ukraine, Carpates

Aconitum firmum Reichenb.

A $325 . \quad$ s.c. 1984 , Suisse, Champex- Lac, HB

Aconitum lamarckii Reichenb.

A 326. R.11987, ab s. n. 1971, Germany, Frankfurt am Main, HBU

Aconitum leucostomum Worosch.

327. s. n. 1971, Kazachstan

Aconitum moldavicum Hacq.

$328 . \quad$ p. n. 1979, Ukraine, Carpates

Aconitum napellus L.

329. p. n. 1979, Ukraine, Carpates

Aconitum raddeanum Regel

330. S. c. 1973 , Rossia, Mosqua, HBP

Aconitum septentrionale Koelle

331. p. n. 1987, Rossia, region Murmansk

Aconitum variegatum $\mathrm{L}$.

332. p. n. 1968, Ukraine, Carpates

Aconitum vulparia Rchb.

333. s. n. 1973, Suisse, Champex-Las, HB

Anemone alpina $\mathrm{L}$.

334.

R. 1 1978, ab s. c. 1957, Czechia, Liberec, HB

Anemone alpina ssp. apiifolia (Scop.) O. Bolos \& Vigo

335.

s. n. 1980, Suisse, Chambesy/Geneve, HB

Anemone narcissiflora L. 
336. s. c. 1956 , France, Grenoble, HB Alp

Anemone narcissiflora L. ssp. fasciculata (L.) Ziman \& Fedor.

337. R. 11967 ab s. n. 1940, Georgia, Bakuriani

Aquilegia amurensis Kom.

338. s. n. 1981, Rossia, Chabarovsk, Arb DalNIILH

Aquilegia atrata Koch

339. s. n. 1982, Germany, Hamburg, HBU

Aquilegia atrovinosa M. Pop. ex Gamajun. I

$340 . \quad$ s. c. 2015 , Rossia, Blagoveschensk, HB

Aquilegia aurea Janka

A $341 . \quad$ s. c. 2001 , Norway, Oslo, HBU

Aquilegia baluchistanica Qurensch et Chaudri
342.
s. c. 2013 , Czechia, PIzen

Aquilegia borodinii Schischk.

A $343 . \quad$ s. n. 1997, Rossia, Krasnojarsk, HBU

Aquilegia brevistyla Hook.

$344 . \quad$ s. n. 1981 , Canada, Vancouver, HBU

Aquilegia caerulea James

345. R. 1 1987, ab p. c. 1981, USA trans Syktyvkar

Aquilegia colchica Kem.-Nath.

$346 . \quad$ s. c. 1981 , Estonia, Tartu, HBU

Aquilegia formosa Fisch.

$347 . \quad$ s. c. 1989 , Canada, Vancouver, HBU

Aquilegia glandulosa Fisch. ex Link

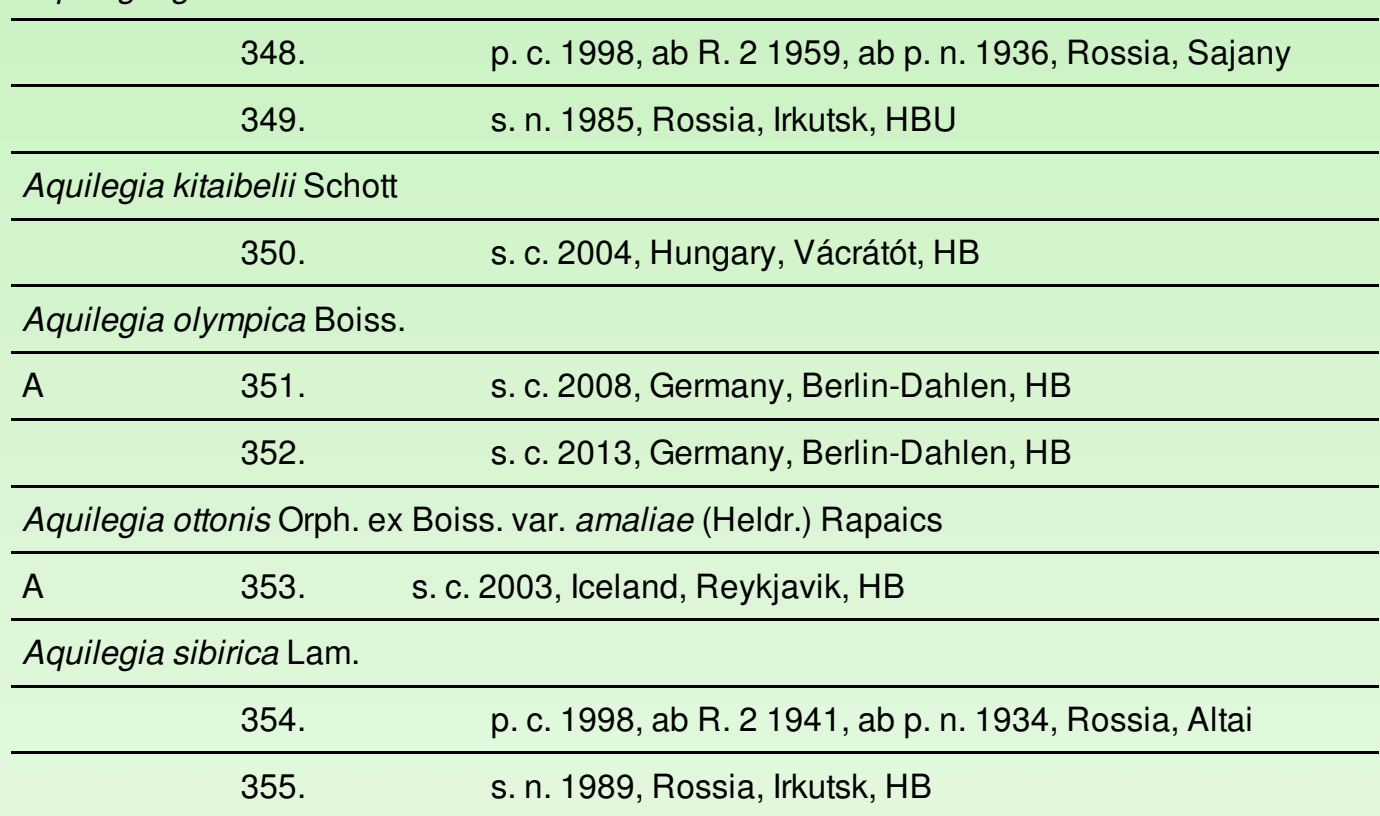

Caltha palustris $\mathrm{L}$. 
$356 . \quad$ p. n. 1979, Ukraine, Carpates

\section{Delphinium cuneatumStev.exDC.}

\begin{tabular}{lll}
\hline A 357. & s. c. 2015 , Rossia, Blagoveschensk, HB
\end{tabular}

Delphinium dictyocarpum DC.

\begin{tabular}{rll}
\hline A & 358. & s. c. 2015, Rossia, Blagoveschensk, HB \\
\hline Delphinium elatum L. & \\
\hline 359. & s. n. 1958, Czechia, Peč \\
\hline 360. & s. n. 1972 , Rossia, Uglich \\
\hline \multirow{2}{*}{361.} & s. c. 1985, Rossia, Novosibirsk, HB \\
\hline 362. & s. n. 1987, Suisse, Basel, HB \\
\hline
\end{tabular}

Delphinium giraldii Diels

\begin{tabular}{lll}
\hline A & 363. & s. c. 2014, Sweden, Göteborg, HB
\end{tabular}

Delphinium grandiflorum L. cv. Summer Blues

\begin{tabular}{lll}
\hline A & 364. & s. c. 2015, Rossia, Blagoveschensk, HB
\end{tabular}

Delphinium oxysepalum Borb. et Pax

$365 . \quad$ s. c. 1982 , Polonia, Zakopane, HB

Delphinium pyramidatum Albov

\begin{tabular}{ll}
\hline 366. & p. n. 1958, Georgia, Bakuriani \\
\hline 367. & R. 21989, ab p. n. 1958, Georgia, Bakuriani \\
\hline 368. & s.n. 1987, Caucasus \\
\hline
\end{tabular}

Helleborus purpurascens Waldst. et Kit.

$369 . \quad$ p. n. 1956, Ukraine, Carpates

Paraquilegia anemoides Ulbr.

\begin{tabular}{|c|c|c|}
\hline * & 370. & s. c. 2005 , Polonia,Poznan, HBU \\
\hline \multicolumn{3}{|c|}{ Thalictrum aquilegifolium $\mathrm{L}$. } \\
\hline A & 371. & p. c. 2008 , ab s. c. 1964 , Romania, Cluj, HBU \\
\hline \multicolumn{3}{|c|}{ Thalictrum aquilegifolium L. var sibiricum Regel \& Tiling } \\
\hline & 372. & s. n. 1987, China, Peking, $\mathrm{HBI}$ \\
\hline
\end{tabular}

Thalictrum minus $\mathrm{L}$.
* 373.
s. n. 1972, Rossia, Narjan-Mar, flora localis

Thalictrum minus L. ssp. kemense (Fr.) Cajander

$374 . \quad$ s. n. 1987, Rossia, Kamtschatka

Thalictrum minus L. ssp. olympicum (Boiss. \& Heldr.) Strid

A $375 . \quad$ s. c. 1998 , Austria, Innsbruck, HBU

Thalictrum uncinnatum Rehm.

* $376 . \quad$ p. n. 1974, Ukraine, Carpates

Thalictrum ussuriense A. Luferov 


\begin{tabular}{|c|c|}
\hline 377. & s. c. 2015 , Rossia, Blagoveschensk, HB \\
\hline \multicolumn{2}{|c|}{ Trollius altaicus C.A. Mey. } \\
\hline 378. & R. 2010, ab s. n. 1953, Rossia, Altai, 4 \\
\hline 379. & s. n. 1984, Kirghizia \\
\hline \multicolumn{2}{|l|}{ Trollius asiaticus L. } \\
\hline 380. & R. 2010, ab p. n. 1934, Rossia, Altai, 4 \\
\hline \multicolumn{2}{|c|}{ Trollius chinensis Bunge } \\
\hline 381. & s. c. 1998 , Germany, Halle, HBU \\
\hline \multicolumn{2}{|c|}{ Trollius europaeus L. ssp. europaeus L. } \\
\hline 382. & s. n. 1958, Czechia, Peč \\
\hline \multicolumn{2}{|l|}{ Trollius ircuticus Sipl. } \\
\hline 383. & s. n. 1989, Rossia, Irkutsk, HB \\
\hline \multicolumn{2}{|c|}{ Trollius ledebourii Rchb. } \\
\hline 384. & R. 1 1960, ab p. n. 1936, Rossia, Sajany \\
\hline \multicolumn{2}{|c|}{ Trollius ranunculinus Stearn } \\
\hline 385. & p. n. 1973, Caucasus \\
\hline 386. & s. c. 1980 , Suisse, Champex-Las, HB \\
\hline
\end{tabular}

ROSACEAE Juss.

Acaena argentea Ruiz. et Pav.

$387 . \quad$ s. c. 1991 , Estonia,Tallinn, HB

Aruncus dioicus (Walt.) Fern.

$388 . \quad$ s. n. 1986, Rossia, Sachalin

Aruncus vulgaris Rafin.

$389 . \quad$ s. c. 1952, Rossia, Mosqua, HBP

Geum $\times$ borisii Kellerer ex Sund.

390.

s. c. 1956 , Czehia, Praha, HB

Geum bulgaricum Pančič

391. S. c. 1980 , Germany, Podsdam, HB

Geum coccineum hort.

\begin{tabular}{ll}
\hline 392. & R. 11951, ab s. c. 1940, Lithuania, Kaunas, HBU \\
\hline 393. & R. 21954, ab s. c., 1940, Lithuania, Kaunas, HBU \\
\hline 394. & s. c., 1954, Rossia, Nizhni Novgorod, HBU \\
\hline 395. & s. c., 1993, Germany, Berlin-Dahlen, HB \\
\hline 396 & s. c., ab s. n. 1994, Germany, Berlin-Dahlen, HB
\end{tabular}

Geum montanum L.

397.

R. 2 1953, ab s. n. 1947, France, Samoëns, HB 


\begin{tabular}{ll}
\hline 398. & R. 21958, ab s. n. 1947, France, Samoëns, HB \\
\hline 399. & R. 11959, ab s. n. 1947, France, Samoëns, HB \\
\hline 400. & p. n. 1970, Ukraine, Carpates \\
\hline 401. & p. n. 1979, Ukraine, Carpates
\end{tabular}

Geum pyrenaicum Mill.
402.
s. n. 1994, Germany, Berlin, HB

Geum quellyon Sweet cv. Georgenberg

A $403 . \quad$ s. n. 2005 , Iceland, Akureyri, HB

Geum rhodopaeum Stoj. et Stef.

404. S. c. 1957 , Czechia, Liberec, HB

Geum rivale L.

A $405 . \quad$ s. c. 2008 , Austria, Salzburg, HBU

Geum virginianum $\mathrm{L}$.

406. S. c. 1956, Czehia, Praha, HB

Potentilla argentea L.

407.

s. n. 2008 , Italia, Aosta, HB

Potentilla argyrophylla Wall. ex Lehm.

$408 . \quad$ s. c. 1990 , Sweden, Göteborg, HB

Potentilla aurea L.

$409 . \quad$ s. n. 1956, Ukraine, Carpates, 1

410. R. 11981 ab s. n. 1957, Ukraine, Carpates, 1

Potentilla chrysantha Trev.
411.
p. n. 1964, Rossia, Altai

Potentilla dombeyi Nestl.

412. S. n. 2004, Slovakia, Bratislava, HBU

Potentilla hirta L. cv. White Beauty

413.

s. c. 1993 , Hungary, Debrecen, HBU

Potentilla heptaphylla L.

A $414 . \quad$ p. c. ab s. n. 1970, Suisse, Chambesy / Geneve, HB

Potentilla leucopolitana P.J. Mull.

A $415 . \quad$ s. c. 2011 , Romania, Cluj, HBU

Potentilla nepalensis Hook. cv. Miss Willmont

A 416 s. c. 2006 , Bjelorossia, Vitebsk, HBU

Potentilla nivea L.

417.

s. c. 2017 , Rossia, Jakutsk, HB

Potentilla rupestris L.

A $418 . \quad$ s. c. 2009 , Romania, Cluj, HBU




\begin{tabular}{|c|c|}
\hline Potentilla $\times$ suendel & ii hort. \\
\hline 419. & s. c. 1970 , Germany, Jena, HB \\
\hline Sanguisorba stipula & \\
\hline 420. & s. n. 1996, China \\
\hline
\end{tabular}

$$
\text { 421. p. c. } 2014 \text { ab R. } 11973 \text { abs. c. 1939, Rossia, Mosqua, NPO }
$$

\section{SAXIFRAGACEAE Juss.}

Bergenia crassifolia (L.) Fritsch

\begin{tabular}{cl}
\hline 422. & R. ab p. n. 1936, Rossia, Sajany, 3 \\
\hline 423. & s. n. 1975, Rossia, Altai trans Novosibirsk, HB \\
\hline 424. & s. c. 1982, Netherlands, Wageningen, HBU \\
\hline 425. & s. c. 2007 , Hungary, Vácrátót, HB
\end{tabular}

Bergenia crassifolia (L.) Fisch. var. pacifica (Kom.) Kom. ez Nekr.

426. p. n. 1979, Rossia, Oriens extremus

Bergenia pacumbis (Buch.-Ham. ex D. Don) C. Y. Wu \& J. T. Pan.
A
427.
s. c. 2004 , Iceland, Akureyri, HB

Bergenia $\times$ smithii Engl. et Irmsch.

$428 . \quad$ s. c. 1956 , Germany, Stuttgart, HBU

Boykinia aconitifolia Nutt.

$\begin{array}{lll}\text { A } & 429 . & \text { s. c. } 2007 \text {, France, Nancy, HBU }\end{array}$

Heuchera chlorantha Piper

A $430 . \quad$ s. c. 2008 , Germany, Marburg, HBU

Heuchera himalayensis Deche. ex Jacques

A 431 s. c. 2013 , Germany, Freiburg, HBU

Saxifraga repanda Willd. ex Sternb.

432. p. n. 1985, Rossia, Mosqua, HBP

Saxifraga spathularis Brot.

433. s. n. 2000 , Portugal, Porto, HB

SCROPHULARIACEAE Juss.

Lagotis uralensis Schischk.

434. R. 1 1956, ab s. n. 1945, Rossia, Ekaterinburg, HBU

Penstemon procerus Douglas ex Graham

435. S. c. 1951 , Rossia, Sankt-Petersburg, HBIB

Veronica gentianoides Vahl

436. p. n. 1955 , Caucasus, Tschinvali 
437. p. n. 1973, Rossia, Daghestan

\begin{tabular}{|c|c|}
\hline \multicolumn{2}{|l|}{ Veronica longifolia L. } \\
\hline 438. & s. n. 1971, Rossia, Narjan-Mar, flora localis \\
\hline 439. & r. 2000, ab s. n. 1947, Rossia, Igarga \\
\hline \multicolumn{2}{|l|}{ Veronica prostrata L. } \\
\hline 440. & s. c. 2016 , Rossia,Tomsk, HBU \\
\hline \multicolumn{2}{|c|}{ Wulfenia carinthiaca Jacq. } \\
\hline 441. & R.1 1953, ab s. c. 1947, Norway, Bergen, HBU \\
\hline 442. & R.2 1963, ab s. c. 1947, Norway, Bergen, HBU \\
\hline \multicolumn{2}{|l|}{ SOLANACEAE Juss. } \\
\hline \multicolumn{2}{|c|}{ Scopolia carniolica Jacq. } \\
\hline 443. & p. n. 1988, Ukraine, Carpates \\
\hline \multicolumn{2}{|c|}{ VALERIANACEAE Batsch } \\
\hline \multicolumn{2}{|c|}{ Valeriana alliariifolia Adams } \\
\hline 444. & s. n. 1992, Germany, München, HB \\
\hline Valeriana montana L & \\
\hline 445. & s. n. 1988, Suisse \\
\hline
\end{tabular}

Valeriana officinalis L.

446. R. 11943 , ab p. n. 1934, Rossia, regio Leningradensis

447. s. n. 1988, Italia, Bormio, HB

Valeriana sambucifolia Mikan fil.

$448 . \quad$ s. n. 1983 , Norway, Troms $\varnothing, \mathrm{HU}$

Valeriana tiliifolia Troitzk.

449. p. n. 1966, Rossia, Naltchik, HBU

\section{PLANTAE IN CALIDARIS CULTAE}

ACANTHACEAE Juss.

Ruellia brevifolia (Pohl) C. Ezcurra

$450 . \quad$ p.c. 1956

ARACEAE Juss.

Alocasia odora (Lodd.) Spach

451. p. c. 1950 , Rossia, Kirovsk, HB

BROMELIACEAE

Acanthostachys strobilacea (Schult. fil.) Klotzsch et Otto

452. S. c. 1957 , Germany, Frankfurt am Main, HBU

ASPHODELACEAE Juss. 


\begin{tabular}{|c|c|}
\hline Chlorophytum macro & Ium (A. Rich.) Aschers. ex Baker \\
\hline 453. & s. c. 1991 , Finland, Yoensu, HBU \\
\hline Mammillaria proliferc & I.) Haw. \\
\hline 454. & s. c. 1964 , Ukraine, Kiev, HBU \\
\hline Mammillaria prolifere & I.) Haw. ssp. multiceps (Salm-Dyck) U. Guzman \\
\hline 455. & p. c. 1958 , France, Antibes, HB \\
\hline Parodia concinna (M & N. P. Taylor \\
\hline 456. & s. c. 1976 , Germany, Frankfurt am Main, HBU \\
\hline Pfeiffera ianthothele & vv.) Web. \\
\hline 457. & s. c. 1993 , Germany, Düsserldorf, HBU \\
\hline Pseudorhipsalis ram & a (Salm-Dyck) Barthlott \\
\hline 458. & s. c. 1995 , France, Lyon HB \\
\hline Rhipsalis virgata We & \\
\hline 459. & p. c. 1958 , Rossia, Mosqua, HBP \\
\hline
\end{tabular}

\section{SEMINA PLANTARUM SPONTANEARUM REGIONIS MURMANENSIS LECTA}

Signa locorum ubi semina lecta sunt: Apat. - In vicinitate urbis Apatity inter lacum Imandra et montes Chibiny, Plant. - Semina plantarum regionis Murmanensis in Horto nosto Reproductatum, Chib. - Montes Chibiny.

\begin{tabular}{cl}
\hline \multicolumn{2}{l}{ Aconitum septentrionale Koelle } \\
\hline 460. & Plant. \\
\hline 461. & Apat. \\
\hline Allium schoenoprasum L. & \\
\hline 462. & Plant. \\
\hline Antennaria dioica Gaertn. & \\
\hline 463. & Apat. \\
\hline Aster sibiricus L. & \\
\hline 464. & Plant. \\
\hline 465. & Apat. \\
\hline Caltha palustris L. & \\
\hline 466. & Plant. \\
\hline Campanula rotundifolia L. & \\
\hline 467. & Apat. \\
\hline Cirsium heterophyllum (L.) Hill. \\
\hline 468. & Plant. \\
\hline Dianthus superbus L. & \\
\hline 469. & Apat. \\
\hline
\end{tabular}




\begin{tabular}{|c|c|}
\hline \multicolumn{2}{|c|}{ Epilobium hornemanii Reichenb. } \\
\hline 470. & Plant. \\
\hline \multicolumn{2}{|c|}{ Epilobium lactiflorum Haussen. } \\
\hline 471. & Plant. \\
\hline \multicolumn{2}{|c|}{ Erigeron acris L. ssp. politus (Fr.) H. Lindb. } \\
\hline 472. & Apat. \\
\hline \multicolumn{2}{|c|}{ Geranium sylvaticum L. } \\
\hline 473. & Plant. \\
\hline \multicolumn{2}{|l|}{ Geum rivale L. } \\
\hline 474. & Plant. \\
\hline \multicolumn{2}{|c|}{ Hedysarum alpinum L. } \\
\hline 475. & Plant. \\
\hline 476. & Apat. \\
\hline \multicolumn{2}{|c|}{ Hedysarum hedysaroides (L.) Schiz. \& Thell. ssp. arcticum (B. Fedtsch.) P. W. Bal } \\
\hline 477. & Apat. \\
\hline \multicolumn{2}{|c|}{ Helianthemum nummularium (L.) Mill. } \\
\hline 478. & Apat. \\
\hline \multicolumn{2}{|l|}{ Hieracium alpinum $\mathrm{L}$. } \\
\hline 479. & Apat. \\
\hline \multicolumn{2}{|c|}{ Hypericum maculatum Crantz } \\
\hline 480. & Apat. \\
\hline \multicolumn{2}{|c|}{ Lathyrus vernus (L.) Bernh. } \\
\hline 481. & Plant. \\
\hline 482. & Apat. \\
\hline \multicolumn{2}{|c|}{ Leucanthemum vulgare Lam. } \\
\hline 483. & Plant. \\
\hline 484. & Apat., Plant. \\
\hline \multicolumn{2}{|c|}{ Ligularia sibirica (L.) Cass. } \\
\hline 485. & Plant. \\
\hline \multicolumn{2}{|c|}{ Melandrium lapponicum (Simm.) Kuzen. } \\
\hline 486. & Apat. \\
\hline \multicolumn{2}{|c|}{ Myosotis asiatica (Vestergren) Schischk. et Serg. } \\
\hline 487. & Plant. \\
\hline \multicolumn{2}{|c|}{ Myosotis decumbens Host| } \\
\hline 488. & Plant. \\
\hline \multicolumn{2}{|c|}{ Myosotis scorpioides L. f. albiflora } \\
\hline 489. & Plant. \\
\hline
\end{tabular}




\begin{tabular}{|c|c|}
\hline \multicolumn{2}{|c|}{ Omalotheca norvegica (Gunn.) Sch. Bip. et F. Schultz } \\
\hline 490. & Plant. \\
\hline \multicolumn{2}{|l|}{ Paeonia anomala L. } \\
\hline 491. & Plant. \\
\hline 492. & Apat. \\
\hline \multicolumn{2}{|c|}{$\begin{array}{l}\text { Pilosella peleteriana (Mérat) F. W. Schultz \& Sch. Bip. ssp. subpeleteriana (Nägeli \& } \\
\text { Peter) P. D. Sell }\end{array}$} \\
\hline 493. & Plant. \\
\hline 494. & Apat. \\
\hline \multicolumn{2}{|l|}{ Phleum alpinum $\mathrm{L}$. } \\
\hline 495. & Plant. \\
\hline \multicolumn{2}{|c|}{ Polemonium caeruleum L. } \\
\hline 496. & Apat. \\
\hline \multicolumn{2}{|c|}{ Potentilla crantzii (Crantz) G. Beck et Fritsch } \\
\hline 497. & Plant. \\
\hline 498. & Apat. \\
\hline \multicolumn{2}{|c|}{ Sedum roseum (L.) Scop. } \\
\hline 499. & Plant. \\
\hline 500. & Apat. \\
\hline \multicolumn{2}{|c|}{ Sibbaldia procumbens L. } \\
\hline 501. & Plant. \\
\hline 502. & Apat. \\
\hline \multicolumn{2}{|c|}{ Solidago virgaurea L. ssp. lapponica (With.) Tzvelev } \\
\hline 503. & Apat. \\
\hline \multicolumn{2}{|c|}{ Silene suecica (Lodd.) Greuter \& Burdet } \\
\hline 504. & Plant. \\
\hline 505. & Apat. \\
\hline \multicolumn{2}{|l|}{ Tanacetum vulgare $\mathrm{L}$. } \\
\hline 506. & Apat. \\
\hline \multicolumn{2}{|c|}{ Tripleurospermum hookeri Sch. Bip. } \\
\hline 507. & Plant. \\
\hline 508. & Apat. \\
\hline \multicolumn{2}{|c|}{ Tofieldia pusilla (Michx.) Pers. } \\
\hline 509. & Chib. \\
\hline \multicolumn{2}{|l|}{ Trollius europaeus L. } \\
\hline 510. & Plant. \\
\hline
\end{tabular}




\begin{aligned} & \hline 511. Plant. \\ & \hline 512. Apat. \\ & \hline Veronica longifolia L. \\ & \hline 513. Plant. \\ & \hline Viola montana L \\ & \hline 514. Apat. \\ & \hline\end{aligned}

\section{N. A. AVRORIN POLAR ALPINE BOTANICAL GARDEN ET INSTITUTE}

\section{KOLA SCIENSE CENTRE}

RUSSIAN ACADEMY OF SCI

184209 APATITY

Academgorodok, 18-A

Murmansk region

E-mail: tnn_aprec@mail.ru

ПОЛЯРНО-АЛЬПИЙСКИЙ БОТАНИЧЕСКИЙ САД

ИМЕНИ Н. А. АВРОРИНА КОЛЬСКОГО НАУЧНОГО ЦЕНТРА РОССИЙСКОЙ АКАДЕМИИ HAYK

184209, г. АПАТИТЫ

Академгородок, д. 18-А Мурманской области

E-mail:tnn_aprec@mail.ru

\section{Литература}

Takhtadzhyan A. L. Sistema magnoliefitov . St. Petersburg, 1987. S. 338.

Cherepanov S. K. Sosudistye rasteniya Rossii i sopredelnich gosudarstv . St. Petersburg, 1995. S. $586-587$.

The Plant List, 2013. Version 1.1; URL: http://www.theplantlist.org/ (accessed on: 28.04.2018). 


\section{Catalogue of seeds of the Polar-Alpine Botanical Garden- Institute № 69}

\begin{tabular}{|c|c|}
\hline $\begin{array}{l}\text { TROSTENYUK } \\
\text { Nadezhda Nikolaevna }\end{array}$ & $\begin{array}{l}\text { Polar-Alpine Botanical Garden-Institute, } \\
\text { Fersman st., 18A, Apatity, 184209, Russia } \\
\text { tnn_aprec@mail.ru }\end{array}$ \\
\hline $\begin{array}{l}\text { VIRACHEVA } \\
\text { Ljubov Leonidovna }\end{array}$ & $\begin{array}{l}\text { Polar-Alpine Botanical Garden-Institute, } \\
\text { Fersman st., 18A, Apatity, 184209, Russia } \\
\text { viracheva-ljubov@yandex.ru }\end{array}$ \\
\hline $\begin{array}{l}\text { GONCHAROVA } \\
\text { Oxana Alexandrovna }\end{array}$ & $\begin{array}{l}\text { Polar-Alpine Botanical Garden, } \\
\text { Fersman st., 18A, Apatity, 184209, Russia } \\
\text { goncharovaoa@mail.ru }\end{array}$ \\
\hline $\begin{array}{l}\text { KIRILLOVA } \\
\text { Natalia Ruslanovna }\end{array}$ & $\begin{array}{l}\text { Polar-Alpine Botanical Garden-Institute a, } \\
\text { Fersman st., 18A, Apatity, 184209, Russia } \\
\text { knr81@mail.ru }\end{array}$ \\
\hline $\begin{array}{l}\text { LIPPONEN } \\
\text { Irina Nikolaevna }\end{array}$ & $\begin{array}{l}\text { Polar-Alpine Botanical Garden-Institute a, } \\
\text { Fersman st., 18A, Apatity, 184209, Russia } \\
\text { lipponen-in@yandex.ru }\end{array}$ \\
\hline
\end{tabular}

\section{Key words:}

seed list, Polar-Alpine Botanical Garden-Institute, PABGI, seeds, introduction, Index seminum
Summary: A list of seeds of cultivated plants collected in 20172018 at the Polar-Alpine Botanical Garden-Institute, offered for exchange with other botanical gardens and institutions.

Is received: 07 may 2019 year

Is passed for the press: 31 july 2019 year

\section{References}

Cherepanov S. K. Vascular plants of Russia and neighboring countries. St. Petersburg, 1995. S. $586-587$.

Takhtadzhyan A. L. Magnoliefite system. St. Petersburg, 1987. S. 338.

The Plant List, 2013. Version 1.1; URL: http://www.theplantlist.org/ (accessed on: 28.04.2018).

Цитирование: Тростенюк Н. Н., Вирачева Л. Л., Гончарова О. А., Кириллова Н. Р., Липпонен И. Н. Каталог семян Полярно-Альпийского ботанического сада-института № 69 // Hortus bot. 2019. T. 14, 2019, стр. 584 - 616, URL: http://hb.karelia.ru/journal/atricle.php?id=6345.

DOI: $10.15393 / \mathrm{j} 4$. art.2019.6345

Cited as: Trostenyuk N. N., Viracheva L. L., Goncharova O. A., Kirillova N. R., Lipponen I. N. (2019). Catalogue of seeds of the Polar-Alpine Botanical Garden-Institute № 69 // Hortus bot. 14, 584 - 616. URL: http://hb.karelia.ru/journal/atricle.php?id=6345 